\title{
State reversals of optically induced tilt and torsional eye movements
}

\author{
RONALD A. FINKE and RICHARD HELD \\ Massachusetts Institute of Technology, Cambridge, Massachusetts 02139
}

\begin{abstract}
Alternations of the state of apparent self-motion during observation of a large visual display rotating about the line of sight are associated with alternations in the magnitude of induced tilt and torsional eye rotation. In one experiment, shifts in visually induced tilt during these state alternations are found to be in the opposite direction to corresponding shifts in induced ocular torsion. In a second experiment, the reversals of self-motion perception are shown to be an intravisual phenomenon, independent of competing inputs provided by the vestibular system. These results emphasize the importance of distinguishing between visual and vestibular processes in tilt perception and ocular rotation during human orientation to gravitational vertical.
\end{abstract}

The perception of bodily movement and orientation in space is provided by information from the visual and vestibular systems. These two systems appear to interact in complementary fashion, with perception of rapid acceleration provided mainly by the vestibular system and perception of slower acceleration and constant velocity provided by the visual system. For example, such findings have been provided by psychophysical studies on visually induced sensations of rotation, termed circular vection (e.g., Dichgans \& Brandt, 1973; Young, Dichgans, Murphy, \& Brandt, 1973), and by similar studies on visually induced sensations of rectilinear movement, termed linearvection (e.g., Berthoz, Pavard, \& Young, 1975). On the basis of neurophysiological evidence, it has been argued that the vestibular nucleus receives direct influences from visual stimulation in response to moving optical patterns, corresponding to sensations of rotary and linear movement (Dichgans, Schmidt, \& Graf, 1973; Henn, Young, \& Finley, 1974; Daunton $\&$ Thomsen, Note 1). It has also been shown that large visual displays rotating about an observer's line of sight induce sensations of tilt, in the opposite direction to field rotation, of approximately $10^{\circ}-15^{\circ}$ from gravitational vertical (Dichgans, Held, Young, \& Brandt, 1972; Held, Dichgans, \& Bauer, 1975). The visually induced tilt is apparently constrained by veridical input from the otolith organs, resulting in a paradoxical sensation of continuous motion at a constant tilt.

Observation of a visual field rotating about the line of sight also induces rotary eye movements in

This research was supported by NASA Grant NGL 22-009-308 and in part by Grant NIH 5-RO1-EYO1191. Requests for reprints should be addressed to Ronald A. Finke, Department of Psychology, E10-114A, Massachusetts Institute of Technology, 79 Amherst Street, Cambridge, Massachusetts 02139. the same direction as the moving field, referred to as ocular torsion. Visually induced eye torsion is typically on the order of $1^{\circ}-2^{\circ}$ (e.g., Dichgans et al., 1972), while eye torsion produced by head tilt (ocular counterrolling) may be as large as $6^{\circ}-8^{\circ}$ (Miller, 1961). Since it has been assumed that tilt perception and ocular torsion result from the same vestibular processes (see Howard \& Templeton, 1966), visually induced torsion is sometimes taken as the cause of visually induced tilt (e.g., Hughes, Brecher, \& Fishkin, 1972). Merker and Held (Note 2) recently confirmed that the visually induced sensation of tilt is increased as a function of head tilt (see also Dichgans, Diener, \& Brandt, 1974; Young, Oman, \& Dichgans, 1975), allegedly because of the decrease in efficiency of the otoliths (Howard \& Templeton, 1966; Young, 1974). However, Merker and Held also found that visually induced torsion is not increased as a result of head tilt, implying that this torsion is independent of the vestibular constraints on induced tilt perception. For rotation of the visual field about an observer's vertica! axis, Brandt, Dichgans, and Büchele (1974) found that induced sensations of circularvection were not correlated with optokinetic nystagmus, and that these two effects could be dissociated. These findings suggest a general dissociation between oculomotor and perceptual mechanisms; in the present study, two experiments are reported which further demonstrate that visually induced tilt and torsion are mediated by independent processes.

Our method of investigation was to measure tilt and torsion induced in two alternating conditions of self-motion perception, first described in detail by Held et al. (1975). Initially, when an observer begins to view a rotating visual field moving about the line of sight, a sensation of bodily rotation in the opposite direction is produced. After $30 \mathrm{sec}$ to $1 \mathrm{~min}$ of continuous viewing, this sensation of movement 
abruptly ceases, with the observer then feeling stationary, experiencing an increase in the apparent velocity of the rotating display. This condition or state is then replaced with that in which bodily movement is again perceived. During prolonged viewing, these two states alternate more frequently, with an increased period of the state of no apparent bodily motion. Initial observations by Held et al. (1975) of visually induced tilt in this state suggested that the tilt may occasionally be reduced to zero. In the first experiment, we made quantitative measures of tilt and torsion in each state, to determine whether these two effects covaried across conditions of selfmotion perception.

\section{EXPERIMENT 1}

\section{Method}

Subjects. Six male and six female undergraduate students at M.I.T. served as paid observers in the experiment.

Procedure. The experimental apparatus for producing the rotating visual display and measuring the induced tilt has been described in detail previously (see Held et al., 1975). The method for measuring induced tilt involved setting a bar mounted on a target disk to apparent horizontal. Ocular torsion was measured by adjusting the same bar to an orientation that coincided with a bar-shaped afterimage produced by a strobe flash unit, before and again after the initiation of rotation of the visual field. All measures consisted of averaging together five consecutive judgments. Before each measure, the experimenter displaced the target bar slightly, in a randomly chosen direction, to insure that subjects did not simply memorize hand positions on the adjustment knob as a basis for judgments. This procedure also controlled against possible torsional movements induced by rotation of the target bar, since Balliet and Nakayama (Note 3 ) have shown that visual feedback can be used to voluntarily control torsion. Each observer, with head fixed in position using a biteboard, sat upright in front of a large unpatterned disk, which contained random visual noise and subtended $122^{\circ}$ of visual angle. This disk could be rotated in clockwise and counterclockwise directions at a speed of $40^{\circ} / \mathrm{sec}$. The target bar was located at the center of this disk and was mounted on a white circular background subtending $16^{\circ}$.

To facilitate the discrimination of state alternations, a stationary, three-prong sectored field was mounted on a transparent background directly in front of the display field. The sectored field contained $60^{\circ}$ sectors of black alternating with $60^{\circ}$ transparent sectors, with a $16^{\circ}$ transparent central region to permit viewing of the target disk. The three black sectored fields mounted in this manner extended out $21^{\circ}$ of visual angle from the center of rotation. At the beginning of the experiment, the subjects were instructed in the procedure for making horizontal and afterimage measures, and the distinction between the two states was explained. State I was designated as the state in which an observer feels a sensation of apparent bodily rotation, as indicated visually by the apparent rotation of the three-prong sectored field. State 2 was designated as the state in which an observer feels no apparent movement, as indicated by the absence of movement in the sectored field. Thus, the sectored field is seen as a visual extension of the observer's body, just as would his arm if held stationary in front of his eyes. Each observer was permitted to observe these changes in the appearance of the sectored field during state alternations before measures were taken, and all 12 subjects reported that the state reversals could be clearly discriminated. It is important to emphasize that the criterion for judging state conditions involved only the appearance of the sectored field and corresponding bodily sensations of movement; no reference was made to possible changes in tilt or the orientation of the afterimage.

Measures of the apparent horizontal position of the target were recorded first, followed by measures of ocular torsion. At the beginning of this procedure, baseline settings to the horizontal were measured and recorded without field rotation. The field was then rotated in either a clockwise or a counterclockwise direction, and after viewing this rotating field for $30 \mathrm{sec}$, the observer was asked to give judgments of perceived horizontal in each of the two states. The experimenter first requested a horizontal reading in State 1, followed by a reading in State 2, until five pairs of readings were obtained. Occasionally, this required some waiting until the appropriate state reversal occurred. A 1-min rest period was then provided; afterwards, a new baseline was taken and the procedure was repeated for the other direction of rotation. Measures of torsional eye movements were obtained in a similar manner; baseline and rotation settings of eye position were obtained by orienting the target edge to the afterimage bar. The order of clockwise and counterclockwise directions of field rotation was counterbalanced. Tilt and torsion differences between baseline and rotation conditions were calculated separately for each state and each direction of rotation.

\section{Results}

All observers reported that State 1 dominated initially, with State 2 becoming more frequent with continued observation. The period of state alternations ranged from several seconds to $1 / 2 \mathrm{~min}$, and appeared to change spontaneously. Nearly all of the measures showed consistent within-subject differences for the two directions of field rotation, as had been observed previously (e.g., Dichgans et al., 1972; Held et al., 1975). However, since no significant differences were found between subjects on any of the measures for direction of field rotation or between males and females, the results are expressed as the mean total angular shift from baseline levels. In reporting these results, the "positive" direction corresponds to tilt in the direction opposite to that of the rotating visual field, and to torsion in the same direction as the field. In State 1 , the mean tilt of $9.4^{\circ}$ was significantly greater than the tilt of $7.4^{\circ}$ in State 2 (correlated $\mathrm{t}=4.25, \mathrm{df}=11, \mathrm{p}<.01$ ). This difference was actually less than expected based on earlier qualitative observations. The magnitude of induced tilt ranged from $0.1^{\circ}$ to $15.4^{\circ}$ in State 1 and from $-0.4^{\circ}$ to $12.6^{\circ}$ in State 2 . By contrast, the mean torsion of $1.6^{\circ}$ found in State 1 was significantly smaller than the torsion of $3.3^{\circ}$ found in State 2 (correlated $t=3.80$, df $=11, p<.01$ ). The magnitude of induced torsion ranged from $0.1^{\circ}$ to $5.1^{\circ}$ in State 1 , and from $1.7^{\circ}$ to $8.9^{\circ}$ in State 2. All 12 of the observers showed a greater tilt in State 1, while 11 observers showed greater torsion in State 2. Within each state, correlations between induced tilt and torsion were computed and found to be nonsignificant. Although large individual differences were obtained for both measures, differences between the two states were relatively consistent across all observers. 


\section{Discussion}

The results of this experiment show that the magnitudes of visually induced tilt and ocular torsion shift in opposite directions across state reversals of apparent motion. Furthermore, it is clear that torsion is not related in any simple way to sensations of induced tilt. Since the results of Merker and Held (Note 2) had suggested that the gravitational reference sensed by the vestibular system constrains induced tilt but not torsion, it remained to determine whether the state reversals result from an alternate doninance of either vestibular or visual influences. That is, it is of interest to determine whether these state reversals involve a dynamic interaction between visual and vestibular afference, or whether they occur entirely within the visual system. In the latter case, one should expect that state differences in torsion would be independent of vestibular stimulation. To investigate this issue, Experiment 1 was repeated in the supine position, removing vestibular constraints normally provided by the otoliths.

\section{EXPERIMENT 2}

\section{Method}

Subjects. Six of the observers in Experiment 1, four males and two females, returned for an additional paid 1-h session.

Procedure. The procedure was identical to that in Experiment 1 , except that the visual display apparatus was turned $90^{\circ}$ in such a manner that it could be observed in the supine position. Since observers had no gravitational reference for making "horizontal" judgments in this condition, they were instructed to align the target bar in such a manner that it appeared perpendicular to the axis of the head. Previous work by Rock (1954) has shown that observers in the supine position can make such judgments with considerable reliability. Torsional measures were obtained in the usual manner.

\section{Results}

All observers reported that state alternations were present in this condition, appearing equivalent to those observed in the upright position. However, the paradoxical combination of induced bodily motion and constant tilt experienced in State 1 of the previous experiment was removed for State 1 occurring in the supine position. For this condition, all observers experienced unambiguous and continuous motion about the vertical axis. One of the six observers showed extremely large variability $\left(14.5^{\circ}\right)$ in his horizontal judgments, and was excluded from statistical analyses on this measure. In State 1 , the mean horizontal shift of $2.2^{\circ}$ was not significantly different from that of $2.5^{\circ}$ in State 2 (correlated $t=0.37$, $\mathrm{df}=4$, n.s.). These measures ranged from $-4.3^{\circ}$ to $6.9^{\circ}$ in State 1 and from $-2.9^{\circ}$ to $4.0^{\circ}$ in State 2 . Neither the State 1 nor the State 2 horizontal shifts were significantly different from initial reference settings (correlated $t=1.23$, df $=4$, n.s.; corre- lated $\mathrm{t}=1.87, \mathrm{df}=4, \mathrm{n}$.s., respectively). The mean shifts in horizontal judgments were greater in State 1 for only one of the five observers. For torsion, the mean shift of $1.2^{\circ}$ in State 1 was again significantly smaller than that of $2.2^{\circ}$ in State 2 (correlated $t$ $=5.62$, df $=5, \mathrm{p}<.001$ ), with all six observers showing greater torsion in State 2. Measures of torsion ranged from $-0.5^{\circ}$ to $3.5^{\circ}$ in State 1 and from $0.6^{\circ}$ to $4.9^{\circ}$ in State 2 . The State 1 torsion was not significantly different from initial reference settings (correlated $\mathrm{t}=1.84, \mathrm{df}=5$, n.s.), in contrast to the State 2 torsion (correlated $\mathrm{t}=3.53$, $\mathrm{df}=5, \mathrm{p}<.01$ ). For all observers, there were no significant differences in torsion between the upright and supine conditions for either state, while significantly greater tilt was obtained in the upright condition, both for State 1 (correlated $t=5.50$, df $=4, p<.01$ ) and State 2 (correlated $t=3.04$, $d f=4, p<.05$ ). For comparison, the results of both experiments are presented in Figure 1.

\section{Discussion}

Even apart from the measured effects, Experiment 2 shows that the locus of state reversals of apparent bodily motion is intravisual, since the reversals are still present when vestibular constraints for tilt are removed. This result is in agreement with the intravisual "motion habituation" model proposed by Brandt, et al. (1974) to account for similar alternations of self-motion occurring during circularvection about the vertical axis. Further, the results of Experiment 2 show that state differences in torsion appear to be the same in both the upright and supine viewing conditions, demonstrating an intravisual locus for visually induced torsion. In contrast, state differences in visually induced tilt appear only when vestibular constraints are imposed.

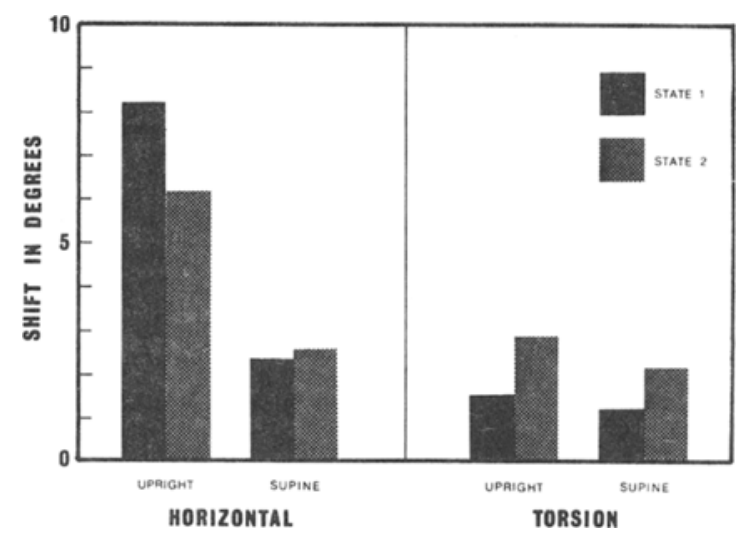

Figure 1. Mean shifts in visually induced tilt and ocular torsion as a function of state alternation and observer position. 


\section{CONCLUSIONS}

The results of these experiments argue for the existence of relatively independent processes underlying visually induced tilt and ocular torsion during observation of rotating visual fields about the line of sight, and they imply that measures of torsion cannot be regarded as accurate indicators of visually induced changes in perceived orientation. These findings are also in support of the current distinction between the "two visual systems" for form and orientation perception (e.g., see Held, 1970; Schneider, 1969). If one regards the function of torsion as facilitating the tracking of objects during head tilt (see Howard \& Templeton, 1966), the visual input for orientation perception (in the form of induced tilt) is dissociated from that for object tracking, with the former interacting with the vestibular system and the latter occurring entirely within the visual system.

\section{REFERENCE NOTES}

1. Daunton, N. C., \& Thomsen, D. D. Otolith-visual interactions in single units of cat vestibular nuclei. Paper presented at the meeting of the Society for Neuroscience, Toronto, November 1976.

2. Merker, B., \& Held, R. Comparison of eye torsion with the apparent horizontal under head tilt and visual field rotation. Paper presented at the meeting of the Eastern Psychological Association, Boston, April 1977.

3. Balliet, R., \& Nakayama, K. Human voluntary torsion and apparent failure of local sign. Paper presented at the meeting of the Association for Research in Vision and Ophthalmology, Sarasota, Florida, April 1976.

\section{REFERENCES}

Berthoz, A., Pavard, B., \& Young, L. R. Perception of linear horizontal self-motion induced by peripheral vision (linearvection): Basic characteristics and visual-vestibular interactions. Experimental Brain Research, 1975, 23, 471-489.

Brandt, T., Dichgans, J., \& Büchele, W. Motion habituation: Inverted self-motion perception and optokinetic after-nystagmus. Experimental Brain Research, 1974, 21, 337-352.
Dichgans, J., \& Brandt, T. The psychophysics of visuallyinduced perception of self-motion. In F. O. Schmidt \& F. G. Worden (Eds.), The neurosciences: Third study program. Cambridge, Mass: M.I.T. Press, 1973.

Dichgans, J., Diener, H. C., \& Brandt, T. Optokineticgraviceptive interaction in different head positions. Acta Otolaryngologica, 1974, 78, 391-398.

Dichgans, J., Held, R., Young, L. R., \& Brandt, T. Moving visual scenes influence the apparent direction of gravity. Science, 1972, 138, 1217-1219.

Dichgans, J., Schmidt, C. L., \& GRaf, W. Visual input improves the speedometer function of the vestibular nuclei in goldfish. Experimental Brain Research, 1973, 18, 319-322.

HELD, R. Two modes of processing spatially distributed visual stimulation. In F. O. Schmidt (Ed.), The neurosciences: Second study program. New York: Rockefeller University Press, 1970.

Held, R., Dichgans, J., \& Bauer, J. Characteristics of moving visual scenes influencing spatial orientation. Vision Research, $1975,15,357-365$.

Henn, V., Young, L. R., \& Finley, C. Vestibular nucleus units in alert monkeys are also influenced by moving visual fields. Brain Research, 1974, 71, 144-149.

HowARD, I. P., \& Templeton, W. B. Human spatial orientation. New York: Wiley, 1966.

Hughes, P. C. Brecher, G. A., \& Fishkin, S. M. Effects of rotating backgrounds upon the perception of verticality. Perception \& Psychophysics, 1972, 11, 135-138.

MILLER, E. F., II. Counterrolling of the human eyes produced by head tilt with respect to gravity. Acta Otolaryngologica, 1961, 54, 479-501.

Rock, I. The perception of the egocentric orientation of a line. Journal of Experimental Psychology, 1954, 48, 367-374.

SCHNeider, G. E. Two visual systems. Science, 1969, 163, 895-902.

YounG, L. R. The role of the vestibular system in posture and movement. In V. B. Mountcastle (Ed.), Medical physiology. St. Louis: Mosby, 1974.

Young, L. R., Dichgans, J., Murphy, R., \& Brandt, T. Interaction of optokinetic and vestibular stimuli in motion perception. Acta Otolaryngologica, 1973, 76, 24-31.

Young, L. R., Oman, C. M.. \& Dichgans, J. Influence of head orientation on visually-induced pitch and roll sensations. Aviation, Space, and Environmental Medicine, 1975, 46, 264-268.

(Received for publication September 16, 1977; revision accepted February 6, 1978.) 